\title{
Comparison of Oral versus Intravenous Proton Pump Inhibitors in Preventing Re-bleeding from Peptic Ulcer after Successful Endoscopic Therapy
}

\author{
Seyed Mohammad Valizadeh Toosi ${ }^{1}$, Ahmad Reza Elahi Vahed ${ }^{2}$, \\ Iradj Maleki ${ }^{3, *}$, Zohreh Bari ${ }^{1}$
}

1. Assistant Professor of Gastroenterology, Gut and Liver Research Center, Mazandaran University of Medical Sciences, Sari, Iran

2. Resident of Internal Medicine, Mazandaran University of Medical Sciences, Sari, Iran

3. Associate Professor of Gastroenterology, Gut and Liver Research Center, Mazandaran University of Medical Sciences, Sari, Iran

\section{* Corresponding Author:}

Iradj Maleki, M.D

Associate Professor of Gastroenterology, Gut and Liver Research Center, Mazandaran University of Medical Sciences, Sari, Iran Telefax: + 981133377176

Email: iradj2001@yahoo.com

Received: 15 Mar. 2018

Accepted: 18 Jul. 2018

\section{ABSTRACT}

\section{BACKGROUND}

Proton pump inhibitors (PPIs) are now widely prescribed for the management of patients with acute upper gastrointestinal bleeding; although its optimal dose and route of administration has remained a controversial issue. The aim of this study was to assess the clinical effectiveness of high dose oral versus intravenous (IV) PPI after successful endoscopic therapy in patients with bleeding peptic ulcer disease.

\section{METHODS}

178 patients with active upper gastrointestinal bleeding due to a peptic ulcer with stigmata of high risk for re-bleeding entered the study. After successful endoscopic hemostasis, they were randomized to receive either high dose oral pantoprazole $(80 \mathrm{mg}$ stat and $80 \mathrm{mg}$ twice daily for 3 days) or high dose intravenous pantoprazole ( $80 \mathrm{mg}$ IV infusion within 30 minutes and $8 \mathrm{mg}$ per hour for 3 days). After the 3 rd day, the patients in both groups received oral pantoprazole $40 \mathrm{mg}$ twice daily for one month. The end points were comparing the rate of re-bleeding or mortality, and the need for blood transfusion or surgery during the first month between the two groups.

\section{RESULTS}

There were not significant statistical differences between the two groups in the volume of blood transfusion, mean duration of hospital stay, need to surgery, or mortality rates. However, the rates of re-bleeding were $2.3 \%(2: 88)$ in the IV group and 3.3\% (3:90) in the oral group $(p=0.6)$.

\section{CONCLUSION}

According to our findings, it seems that high dose oral PPI can be a good alternative to high dose IV PPI in patients with bleeding peptic ulcer who are at high risk of re-bleeding. Due to the lower cost and the availability of oral PPIs, their use can be economically much more affordable.

\section{KEYWORDS:}

Proton pump inhibitor, Peptic ulcer, Hemorrhage, Endoscopic therapy

\section{Please cite this paper as:}

Valizadeh Toosi SM, Elahi Vahed AR, Maleki I, Bari Z. Comparison of Oral versus Intravenous Proton Pump Inhibitors in Preventing Re-bleeding from Peptic Ulcer after Successful Endoscopic Therapy. Middle East J Dig Dis 2018;10:236-241. doi: 10.15171/mejdd.2018.116.

\section{INTRODUCTION}

Peptic ulcer disease is the most common cause of upper gastrointestinal bleeding (UGIB), accounting for about $50 \%$ of cases. ${ }^{1,2}$ In a recent review article from Iran peptic ulcer disease was the most common cause of UGIB (30-65\%) and erosive gastro-duodenopathy ranked the second (16-25\%). ${ }^{3}$ It remains a serious medical problem with significant morbidity and mortality. Endoscopic therapy significantly reduces further bleeding, surgery, and mortality in patients with bleeding peptic ulcers and is now recommended as the first hemostatic modality for these patients. ${ }^{4-6}$ However, there is a high risk of 
peptic ulcer re-bleeding in $14-36 \%$ of patients in spite of efficient endoscopic intervention. ${ }^{7,8}$

Gastric acid inhibits clot formation and promotes clot lyses and accordingly, disturbs hemostasis of ulcers in the stomach and duodenum. ${ }^{9}$ Therefore, reduction of gastric acid secretion can prevent ulcer re-bleeding. ${ }^{8}$ Proton pump inhibitors (PPIs) are the drugs that are widely used to reduce gastric acid secretion. Intravenous (IV) and oral pantoprazole with equal dose have similar acid suppression effect. ${ }^{10}$ Compared to standard dose of oral PPI, high dose oral PPI has faster acid suppression ${ }^{11}$ and also high dose IV PPI has faster adequate acid suppression effect (gastric acid $\mathrm{PH}>6$ ) than high dose oral PPI. ${ }^{11,12}$ However, the optimal route, dose, and duration of PPI therapy after endoscopic therapy of a bleeding peptic ulcer remain controversial.

Several controlled trials and meta-analyses have shown the comparable efficacy of IV and oral PPIs in ulcers at high risk of re-bleeding after endoscopic therapy. However, they mostly recommended further studies to confirm the results. ${ }^{13-17}$

In this study, we attempted to evaluate and compare the effects of IV and oral PPIs in preventing re-bleeding from peptic ulcers after successful endoscopic therapy.

\section{MATERIALS AND METHODS}

\section{Design and patients:}

This study was a single center, prospective, randomized trial conducted in a tertiary teaching hospital (Imam Khomeini Hospital, Sari) in Iran. The protocol was approved by the Ethics Committee of Mazandaran University of Medical Sciences and was also registered in Iranian Registry of Clinical Trials (number: IRCT2014082515510N2). Furthermore, a written informed consent was obtained from all subjects.

From June 2014 to May 2015, all adult patients who were admitted to our Gastroenterology Department with symptoms of UGIB, as documented by hematemesis, melena, or hematochezia, were considered to be included in this study. They were evaluated by upper GI endoscopy during the first 24 hours of admission, after hemodynamic stabilization. It should be mentioned that all patients received IV pantoprazole ( $80 \mathrm{mg}$ stat followed by $8 \mathrm{mg}$ infusion per hour) before endoscopic assessment.

Patients older than 18 years with successful endoscopic therapy of high risk ulcers for re-bleeding [defined as spurting bleeding (Forrest IA), oozing bleeding (Forrest IB), non- bleeding visible vessel (Forrest IIA) or adherent clots (Forrest IIB)] were enrolled in the study. ${ }^{18}$ On the other hand, patients with low risk of bleeding from ulcers (clean base ulcer, flat pigmented ulcers), suspicious malignant ulcer, bleeding tendency, uremia, liver cirrhosis, and Mallory Weiss tear were excluded from the study.

Therapeutic endoscopy for patients with high risk peptic ulcer for bleeding (Forrest IA-IIB) were done by injecting up to $40 \mathrm{~mL}$ of epinephrine (diluted 1:10000) around the ulcer crater to stop bleeding and electrocoagulation therapy by Argon Plasma Coagulation (APC) for all patients. Also, a biopsy sample was taken from antrum for evaluating $H$. pylori infection. For patients with unsuccessful endoscopic therapy, an immediate surgery consultation was performed.

The enrolled patients were randomly allocated into two groups using sealed envelopes containing a therapeutic option (either IV or oral pantoprazole). In the oral pantoprazole (Oral-Pan) group, the patients received pantoprazole (Nolpaza, Iranian pharmaceutical company Actoverco) $80 \mathrm{mg}$ orally early after endoscopy and then twice daily for 72 hours. In the IV pantoprazole (IV-Pan) group, the patients received injective pantoprazole (Pepti care, Iranian Razak Drau Company) $80 \mathrm{mg}$, infused during 30 minutes and then $8 \mathrm{mg} /$ hour IV pantoprazole for 72 hours. After the 3rd day, all the patients of both groups received oral pantoprazole $40 \mathrm{mg}$ twice daily.

During the hospital stay, the serum hemoglobin $(\mathrm{Hb})$ was checked every 8 hours. Blood transfusions were performed if $\mathrm{Hb}$ was lower than $7 \mathrm{~g} / \mathrm{dL}$ in young patients or lower than $9 \mathrm{gr} / \mathrm{dL}$ in patients older than 50 years or in patients with history of ischemic heart disease (IHD) or those being in shock. After endoscopic therapy, rebleeding was suspected if hematemesis reappeared or the patient developed orthostatic hypotension, unstable vital signs (systolic blood pressure $<90 \mathrm{mmHg}$, pulse rate $>120 / \mathrm{min}$ ) or $\mathrm{Hb}$ drop $>2 \mathrm{~g} / \mathrm{dL}$ (despite blood transfusion). Patients suspected to re-bleeding underwent urgent endoscopy and if active bleeding, fresh blood, or blood clots were seen, epinephrine injection and APC were performed. Then they again received pantoprazole according to their protocol group. Also, in case of definite cardiac or neurological indications for continuing Aspirin intake, the drug was given to the patients after 24 hours 
Table 1: Demographic and clinical variables of patients at entry to the study

\begin{tabular}{lccc}
\hline Variable & IV PPI group & Oral PPI group & P value \\
\hline Number of patients (\%) & $88(49.4 \%)$ & $90(50.6 \%)$ & 0.9 \\
\hline Mean age (Years) & $60.3(25-89)$ & $58.4(18-100)$ & 0.8 \\
\hline Sex: $\quad$ Male & $49(55.7 \%)$ & $63(70 \%)$ & 0.04 \\
& $39(44.3 \%)$ & $27(30 \%)$ & 0.3 \\
\hline Smoking & $22(25 \%)$ & $29(32.2 \%)$ & 0.2 \\
\hline History of PUD & $14(15.9 \%)$ & $9(10 \%)$ & 0.4 \\
\hline Aspirin or NSAID & $68(77.2 \%)$ & $63(60 \%)$ & 0.3 \\
\hline Clopidogrel & $7(8 \%)$ & $4(4.4 \%)$ & 0.9 \\
\hline Warfarin & $7(8 \%)$ & $2(2.2 \%)$ & 0.3 \\
\hline Melena & $69(77.3 \%)$ & $77(85.6 \%)$ & 0.5 \\
\hline Hematemesis & $51(57.7 \%)$ & $38(42.2 \%)$ & 0.8 \\
\hline Mean initial Hb (gr/dL) & 9 & 8.7 & 0.9 \\
\hline Mean supine BP (mm Hg) & $113 / 72$ & $112 / 70$ & 0.8 \\
\hline Mean sitting BP & $111 / 71$ & $109 / 69.5$ & \\
\hline
\end{tabular}

NSAID: non-steroidal anti-inflammatory drug; Hb: Hemoglobin, BP: Blood pressure; PPI: proton pump inhibitor; PUD: peptic ulcer disease

Table 2: Endoscopic findings of the patients in the two groups

\begin{tabular}{lccc}
\hline EGD Findings & IV-Pan group & Oral-Pan group & P value \\
\hline Gastric ulcer & 39 & 36 & 0.5 \\
\hline Duodenal ulcer & 57 & 59 & 0.9 \\
\hline Adherent clot & $26(29.5 \%)$ & $18(20 \%)$ & 0.14 \\
\hline Oozing & $23(26.1 \%)$ & $33(36.7 \%)$ & 0.13 \\
\hline Non-bleeding visible vessel & $38(42.2 \%)$ & $38(42.2 \%)$ & 0.8 \\
\hline Spurting & $1(1.1 \%)$ & $1(1.1 \%)$ & 0.9 \\
\hline EGD. Esophagastroduodenoscopy & & &
\end{tabular}

of endoscopic treatment.

On the day of discharge, a standard H. pylori eradication regimen followed by oral PPI (pantoprazole 40mg twice daily) was prescribed for patients infected with $H$. pylori, but the rest of the patients were advised to continue just oral PPI for one month. They were all asked to be visited at the end of one month or sooner in case of any problem.

A questionnaire including demographic characteristics, history of previous UGIB, non-steroidal anti-inflammatory drugs (NSAIDs) or Aspirin use, volume of blood transfusion at entry and during hospital stay, the days of hospital stay, endoscopic findings, and the need for re-endoscopy, and surgery, and mortality rates up to one month after discharge were completed for all patients.

Statistical analysis was performed using SPSS software (version 16, Chicago, IL, USA). The descriptive variables such as mean, standard deviations, and frequency were used. Chi square (X2) and $t$ tests were used as appropriate. $P$ value less than 0.05 was considered as statistically significant.

\section{RESULTS}

From June 2014 to May 2015, 376 patients with clinical evidence of UGIB were admitted to our hospital. Upper GI endoscopy was performed for all patients. 178 patients had endoscopic evidence of high risk peptic ulcers for re-bleeding (according to Forrest classification). They underwent therapeutic endoscopy, using diluted adrenaline injection and APC. Also, in two patients, clips were used to control bleeding. These high risk patients were enrolled in the study; 88 patients were randomly allocated to the IV-Pan group and 90 patients were allocated to the oral-Pan group.

The causes of GI bleeding in the remaining 198 patients were esophageal varices, clean-base ulcers, esophageal cancer, Mallory Weiss tearing, gastric cancer, and Dieulafoy's lesion. They were excluded from the study.

All the patients completed the study. 112 patients were men (63\%) and 66 patients (37\%) were women. Other demographic and also clinical and endoscopic data are shown in tables 1 and 2. 
Table 3: Primary and secondary outcomes in the two groups

\begin{tabular}{lccc}
\hline Outcome & IV-Pan group & Oral-Pan group & \multicolumn{1}{c}{ Pvalue } \\
\hline Mortality (\%) & $3(3.4 \%)$ & $1(1.1 \%)$ & 0.3 \\
\hline Re-bleeding (\%) & $4(4.5 \%)$ & $3(3.3 \%)$ & 0.6 \\
\hline Surgery (\%) & $1(1.1 \%)$ & $1(1.1 \%)$ & 0.9 \\
\hline $\begin{array}{l}\text { Volume of blood transfusion } \\
\text { (unit of packed cell) }\end{array}$ & 113 & 117 & 0.8 \\
\hline $\begin{array}{l}\text { Mean duration of hospital stay } \\
\text { (Days) }\end{array}$ & 3.7 & 3.4 & 0.8 \\
\hline Repeated EGD & $5(5.6 \%)$ & $3(3.3 \%)$ & 0.6 \\
\hline EGD: Esophagogastroduodenoscopy & &
\end{tabular}

Seven patients $(3.9 \%)$ re-bled. Four patients were in the IV-Pan group and three were in the Oral-Pan group. Four of the re-bleedings happened during hospital stay and three happened at the $8^{\text {th }}, 8^{\text {th }}$, and $15^{\text {th }}$ day after hospital discharge, respectively. There were no significant differences between the two groups in the rate of re-bleeding, neither during hospital stay, nor after discharge (table 3).

Accordingly, eight patients needed second endoscopy; five were in the IV-Pan group and three were in the oral-Pan group ( $p=0.6$ ). The reason for the repeated endoscopies were re-bleeding in seven patients (as mentioned previously) and second-look endoscopy to assess the quality of the performed injection and APC in one patient.

Two patients underwent surgery during hospital stay. One was in the IV-Pan group and the other was in the Oral-Pan group. The first patient underwent surgery at the first day of hospital admission and the second patient underwent surgery at the second day due to re-bleeding. The reason for surgery was the inability of therapeutic endoscopy to control the bleeding.

Four patients died; three patients were in the IV-Pan and one in the Oral-Pan group, respectively (table 3). Three patients died at presentation due to massive GIB that could not be controlled endoscopically and they died before undergoing surgery. But the 4th patient underwent surgery and died at the $11^{\text {th }}$ day after surgery. All the patients were older than 60 years.

For all the patients oral feeding was started 24-48 hours after successful therapeutic endoscopy and they were discharged from hospital if they had stable vital signs and acceptable hemoglobin levels. After discharge, all the patients were followed up by phone call contacts up to one month to ask about re-bleeding, hospital readmission, blood transfusion, surgery, and mortality.

\section{DISCUSSION}

According to the results of our study, there were no significant differences between the two groups of IV-Pan and Oral-Pan in the rates of re-bleeding and re-endoscopy, duration of hospital stay, the volume of blood transfusion, and rates of surgery and mortality during one month of follow up. There are several other studies that have $\mathrm{s}$ h o w nalmost the same results.

In 2008, Tsai and colleagues conducted a study in which 156 patients with high risk peptic ulcers were divided into two groups to receive either IV PPI or oral PPI for the first 72 hours after therapeutic endoscopy. Afterwards, all the patients received standard doses of oral PPI. The outcomes of re-bleeding, need to transfusion, mortality, surgery, and duration of hospital stay were similar in both groups. ${ }^{19}$

Also, in 2011, Mostaghni and co-workers showed no significant differences in the rate of re-bleeding, duration of hospital stay, and the volume of blood transfusion among 85 patients with high risk peptic ulcer disease who had received either high dose oral omeprazole or IV pantoprazole during the first 72 hours after therapeutic endoscopy. ${ }^{15}$

In 2012, Yen and others evaluated the adverse outcomes of PUD bleeding in 100 patients who had been divided into two groups of high dose IV and oral PPI after therapeutic endoscopy. They showed that duration of hospital stay was shorter in oral PPI group (1.8 days vs. 3.9 days, respectively), but the difference was not statistically significant. Also, other outcomes of GIB including the rates of re-bleeding, surgery, mortality, and volume of transfusion were similar in both groups. ${ }^{14}$

In another single-center, randomized, controlled, double-blind and double-dummy study in 2014, 244 
patients with bleeding PUD, entered the study after therapeutic endoscopy. 118 patients received high dose IV esomeprazole plus oral placebo, and 126 patients received high dose oral esomeprazole plus placebo IV infusion for 72 hours. The patients were followed up for 30 days after index bleeding. According to the results, no difference existed between the two groups in outcomes of re-bleeding, need to blood transfusion, days of hospital stay, and re-endoscopy. However, this study stopped prematurely and therefore, the results of the study are not conclusive for equivalency or non-inferiority of two treatment regimens. ${ }^{16}$

In 2013, Tsoi and colleagues performed a meta-analysis to compare the outcomes of administering oral versus IV PPI after therapeutic endoscopy in patients with high-risk PUDs. Six randomized clinical trials from 2006 to 2011, including 615 patients, (302 patients in oral PPI and 313 patients in IV PPI groups) were evaluated. The outcomes of re-bleeding, volume of blood transfusion, need for surgery, days of hospital stay, and all-cause mortality showed no statistically significant differences between the two groups. ${ }^{17}$

Finally, according to two recent systematic reviews and meta-analyses, both oral and IV PPI can be effectively used after endoscopic treatment of high risk ulcers. ${ }^{20,21}$

Although our results are almost similar to previous studies, our study had a limitation. The endoscopies had been performed by six gastroenterologists. This might have interfered with the same interpretation of the ulcers. However, we used Forrest classification in order to standardize the interpretation of the ulcers. On the other hand, using Iranian brand of pantoprazole is a strong point of our study.

In conclusion, our study showed no statistically significant difference between the two groups of IV or oral PPI in the outcomes of high risk peptic ulcers after therapeutic endoscopy. Therefore, it seems that high dose oral PPI can be a good alternative to high dose IV PPI in ptients with bleeding peptic ulcer disease. Furthermore, due to the lower cost (approximately 30 times) and availability of oral PPI, its use can be economically much more affordable. We suggest further studies to evaluate the effects of different types of oral PPIs on the outcomes of high risk peptic ulcers after therapeutic endoscopy.

\section{ACKNOWLEDGEMENTS}

The authors cheerfully acknowledge the financial support of Mazandaran University of Medical Sciences.

\section{ETHICAL APPROVAL}

There is nothing to be declared.

\section{CONFLICT OF INTEREST}

The authors declare no conflict of interest related to this work.

\section{REFERENCES}

1. Silverstein FE, Gilbert DA, Tedesco FJ, Buenger NK, Persing J. The national ASGE survey on upper gastrointestinal bleeding. I. Study design and baseline data. Gastrointest Endosc 1981;27:73-9.

2. Rockall TA, Logan RF, Devlin HB, Northfield TC. Incidence of and mortality from acute upper gastrointestinal haemorrhage in the United Kingdom. Steering Committee and members of the National Audit of Acute Upper Gastrointestinal Haemorrhage. BMJ 1995;311:222-6. doi:10.1136/bmj.311.6999.222.

3. Masoodi M, Saberifiroozi M. Etiology and outcome of acute gastrointestinal bleeding in iran: a review article. Middle East J Dig Dis 2012;4:193-8.

4. Cook DJ, Guyatt GH, Salena BJ, Laine LA. Endoscopic therapy for acute nonvariceal upper gastrointestinal hemorrhage: a meta-analysis. Gastroenterology 1992;102:13948.

5. Hwang JH, Fisher DA, Ben-Menachem T, Chandrasekhara V, Chathadi K, Decker GA, et al. The role of endoscopy in the management of acute non-variceal upper GI bleeding. Gastrointest Endosc 2012;75:1132-8. doi:10.1016/j. gie.2012.02.033

6. Laine L, McQuaid KR. Endoscopic therapy for bleeding ulcers: an evidence-based approach based on meta-analyses of randomized controlled trials. Clin Gastroenterol Hepatol 2009;7:33-47. doi: 10.1016/j.cgh.2008.08.016.

7. Marmo R, Rotondano G, Piscopo R, Bianco MA, D'Angella R, Cipolletta L. Dual therapy versus monotherapy in the endoscopic treatment of high-risk bleeding ulcers: a meta-analysis of controlled trials. $\mathrm{Am} \mathrm{J}$ Gastroenterol 2007;102:279-89. doi:10.1111/j.15720241.2006.01023.x.

8. Green FW Jr, Kaplan MM, Curtis LE, Levine PH. Effect of acid and pepsin on blood coagulation and platelet aggregation. A possible contributor prolonged gastroduodenal mucosal hemorrhage. Gastroenterology 1978;74:38-43.

9. Cheng HC, Sheu BS. Intravenous proton pump inhibitors for peptic ulcer bleeding: Clinical benefits and limits. World J Gastrointest Endosc 2011;3:49-56. doi:10.4253/ wjge.v3.i3.49. 
10. Hartmann M, Ehrlich A, Fuder H, Luhmann R, Emeklibas S, Timmer W, et al. Equipotent inhibition of gastric acid secretion by equal doses of oral or intravenous pantoprazole. Aliment Pharmacol Ther 1998;12:1027-32. doi:10.1046/j.1365-2036.1998.00406.x.

11. Sachs G, Shin JM, Howden CW. Review article: the clinical pharmacology of proton pump inhibitors. Aliment Pharmacol Ther 2006;23 Suppl 2:2-8. doi:10.1111/j.13652036.2006.02943.x.

12. Laine L, Shah A, Bemanian S. Intragastric $\mathrm{pH}$ with oral vs intravenous bolus plus infusion proton-pump inhibitor therapy in patients with bleeding ulcers. Gastroenterology 2008;134:1836-41. doi:10.1053/j.gastro.2008.03.006.

13. Phulpoto JA, Bhatti ZA, shaikh A. Comparison of oral and intravenous proton pump inhibitor in patients with high risk bleeding peptic ulcers. Rawal Med J 2013;38:7-10.

14. Yen HH, Yang CW, Su WW, Soon MS, Wu SS, Lin HJ. Oral versus intravenous proton pump inhibitors in preventing re-bleeding for patients with peptic ulcer bleeding after successful endoscopic therapy. BMC Gastroenterol 2012;12:66. doi:10.1186/1471-230X-12-66.

15. Mostaghni AA, Hashemi SA, Heydari ST. Comparison of oral and intravenous proton pump inhibitor on patients with high risk bleeding peptic ulcers: a prospective, randomized, controlled clinical trial. Iran Red Crescent Med $J$ 2011;13:458-63.

16. Sung JJ, Suen BY, Wu JC, Lau JY, Ching JY, Lee VW, et al. Effects of intravenous and oral esomeprazole in the prevention of recurrent bleeding from peptic ulcers after endoscopic therapy. Am J Gastroenterol 2014;109:100510. doi:10.1038/ajg.2014.105.

17. Tsoi KK, Hirai HW, Sung JJ. Meta-analysis: comparison of oral vs. intravenous proton pump inhibitors in patients with peptic ulcer bleeding. Aliment Pharmacol Ther 2013;38:721-8. doi:10.1111/apt.12441.

18. Heldwein W, Schreiner J, Pedrazzoli J, Lehnert P. Is the Forrest classification a useful tool for planning endoscopic therapy of bleeding peptic ulcers? Endoscopy 1989;21:258-62. doi:10.1055/s-2007-1010729.

19. Tsai JJ, Hsu YC, Perng CL, Lin HJ. Oral or intravenous proton pump inhibitor in patients with peptic ulcer bleeding after successful endoscopic epinephrine injection. $\mathrm{Br}$ $J$ Clin Pharmacol 2009;67:326-32. doi:10.1111/j.13652125.2008.03359.x.

20. Siau K, Chapman W, Sharma N, Tripathi D, Iqbal T, Bhala N. Management of acute upper gastrointestinal bleeding: an update for the general physician. $J R$ Coll Physicians Edinb 2017;47:218-30. doi:10.4997/JRCPE.2017.303.

21. Jiang M, Chen P, Gao Q. Systematic Review and NetWork Meta-Analysis of Upper Gastrointestinal Hemorrhage Interventions. Cell Physiol Biochem 2016;39:247791. doi:10.1159/000452515. 